\title{
Mécanismes de déformation relative à l'érosion de cavitation
}

\author{
$\begin{array}{ll}\text { M. Maamouri } & \text { A. Karimi }\end{array}$
}

Institut de Génie atomique, Ecole polytechnique fédérale de Lausanne

\section{Introduction}

Lorsqu'un matériau est exposé à l'attaque de cavitation, il subit un type de chargement à caractère d'onde de choc et de pulses de contrainte. Ces pulses sont très localisés; quelques dizaines de $\mu \mathrm{m}$ de diamètre, et répartis d'une manière aléatoire sur la surface exposée de l'échantillon. La durée de ces chocs est de l'ordre de la $\mu$ s et leur amplitude peut atteindre 2-3 GPa (F. Avellan, A. Karimi). Ceci peut induire une rupture locale entourée d'une déformation plastique avec une vitesse de l'ordre de $10^{5}$. $10^{6} \mathrm{~s}^{-1}$. Dans de telles conditions, le mécanisme de déformation peut être différent de ceux observés dans des essais mécaniques conventionnels.

Malgré le nombre impressionnant des publications dans le domaine d'érosion de cavitation, il existe peu d'informations concernant la réponse des matériaux soumis à ce type d'endommagement et les mécanismes par lesquels l'érosion se produit. Cependant, l'application des techniques utilisées couramment dans des études des matériaux comme la microscopie électronique, ou la diffraction des rayons $\mathrm{X}$ ont mis en évidence certains phénomènes caractéristiques de la déformation induite par la cavitation. Parmi eux, l'apparition de micro-macles et la transformation martensitique ont le plus souvent été signalées dans des alliages industriels complexes comme des superalliages (K. Antony, W. Silence), des aciers inoxydables
(C.J. Heathcock et al.) et des bronzes d'aluminium (R. Wright, D. Mikola).

Etant donnée la multiplicité des processus actifs, l'interprétation des mécanismes de déformation dans ces alliages complexes est relativement difficile. C'est pourquoi dans cette étude nous avons choisi le cuivre pur monocristallin afin de mettre en évidence l'effet pulsatoire de la contrainte sur les mécanismes de déformation. Le comportement à l'érosion du cuivre a été l'objet de plusieurs études. Follansbee et al., lors de l'érosion par des particules solides, ont constaté la formation de cellules de dislocations dont l'aspect varie en s'éloignant de la surface érodée. Dans une profondeur de $20-30 \mu \mathrm{m}$ les cellules de 0,4 $0,5 \mu \mathrm{m}$ de taille, décorées de dipôles et de boucles de dislocations, se forment et donnent lieu aux cellules équiaxiales diffuses dans $30-40 \mu \mathrm{m}$ de la surface. D'après l'examen des taches de diffraction en microscopie électronique, la recristallisation dynamique des couches superficielles semble être confirmée sans l'apparition de micromacles ni de cisaillements. Yves et Ruff, au contraire, en étudiant l'effet de la vitesse d'impact dans le taux d'érosion du cuivre, ont observé une très forte apparition de micromacles de déformation au voisinage de la surface pour des vitesses d'impact supérieures à $20 \mathrm{~m} / \mathrm{s}$. Ceci peut expliquer l'écrouissage très élevé de ces échantillons par rapport aux essais à vitesse d'impact inférieure à $20 \mathrm{~m} / \mathrm{s}$, où le maclage ne se produit pas. Il est à noter que l'apparition des macles

\section{Deformation mechanism relating to cavitation erosion.}

Copper single crystals have been exposed to vortex cavitation attack with various erosive aggressivity and the variable exposure time. The progressive displacement of damage and the deformation induced have been examined by scanning and transmission electron microscopy. Hardening inside the plastic zone has been measured by a microhardness technique.

The thickness of the work-hardened layers depends on the cavitation aggressivity and the exposure time. As regards pure copper monocrystals, the deformation extends to a depth of $1.2 \mathrm{~mm}$. The deformation substructure varies along this distance by moving away from the surface. In the first $50 \mu m$, the deformation twinning is the most active mechanism which creates a complex form of micro twin-crystals and with sliding in the next 50-150 $\mathrm{mm}$. In the wider layers, deformation is carried out by sliding which appears in the MET in the form of dislocation cells whose size increases from 0.5 $\mathrm{m}$ at the beginning to 40-50 $\mathrm{mm}$ at the end of the deformed zone. 



1. Evolution de l'état de surface d'un monocristal de cuivre exposé à l'attaque de cavitation vortex. Temps d'exposition: a) $1,5 \mathrm{~h}$, b) $24 \mathrm{~h}, \mathrm{c}) 106 \mathrm{~h}$, d) $59 \mathrm{~h}$. de déformations dans le cuivre a été signalée également dans d'autres types de sollicitations à très basse température. Blewitt et al., ont effectué des essais de traction sur $\mathrm{Cu} 99,999 \%$ à $4,2-77,3^{\circ} \mathrm{K}$ et ont constaté que la déformation se produit par le glissement discontinu de paquets de bandes qui, dans certaines orientations cristallographiques favorables, est suivi du maclage. Dans une étude intensive en microscopie électronique, Mahajan et al., ont rapporté la nucléation de micro-macles dans des échantillons de cuivre irradiés par des neutrons et vieilli $1 \mathrm{~h}$ à $450^{\circ} \mathrm{C}$.

Dans cette étude nous allons faire des observations systématiques des microstructures de déformation induites par l'attaque de cavitation pour mettre en évidence les effets de la vitesse et de l'accumulation répétée des impacts dus aux collapses.

\section{Procédés expérimentaux}

Les échantillons cylindriques de diamètre $12 \mathrm{~mm}$ et de longueur $17 \mathrm{~mm}$ ont été prélevés de barreaux de cuivre monocristallin orienté selon les directions $<111\rangle$, $<110>$ et $<100>$. Les surfaces exposées ont été polies mécaniquement jusqu'au diamant de $0,4 \mu \mathrm{m}$ avant de subir un polissage électrochimique dans une solution de $\mathrm{H}_{3} \mathrm{PO}_{4}$ diluée dans du méthanol.

Les essais d'érosion ont été effectués dans un générateur de cavitation vortex dit veine tourbillon. Le principe de fonctionnement et les paramètres caractéristiques de la machine ont été expliqués dans une autre publication (A. Karimi, F. Avellan). Il s'agit d'un circuit hydraulique fermé ayant une chambre de cavitation dans laquelle l'écoulement reste rotationnel et un vortex de cavités de vapeur peut se produire par l'émission d'une onde de dépression à l'entrée de la chambre. L'implosion de ces cavités sur la surface de l'échantillon, étant installé perpendiculairement à l'axe du vortex, peut provoquer la déformation et, à long terme, l'érosion des matériaux.

Afin d'observer d'une manière systématique l'évolution structurale qui précède l'érosion, des essais à des temps d'exposition variables allant de $1 \mathrm{~min}$ jusqu'à $100 \mathrm{~h}$ ont été faits. Dans chaque cas les échantillons ont été examinés en microscope électronique à balayage (MEB) et à transmission (MET). Les observations en MET nécessitent un dépôt préalable de cuivre, de 1-2 mm d'épaisseur, sur la surface érodée pour pouvoir amincir les couches superficielles qui contrôlent le comportement à l'érosion des matériaux.

\section{Evolution de l'état de surface}

Les pulses de pression dus à l'implosion des cavités atteignent la surface d'explosion de $\sim 12 \mathrm{~mm}^{2}$ de superficie d'une manière aléatoire. L'amplitude de ces pulses est aussi variable et dépende d'une part des conditions hydrodynamiques au voisinage du collapse et d'autre part de la distance entre le centre du collapse et la surface de l'échan- 
tillon. F. Avellan et A. Karimi, en mesurant la vitesse de déplacement de paroi de cavité et la vitesse d'onde de choc émise après l'implosion, ont pu estimer une surpression de l'ordre de 2-3 GPa à l'endroit du collapse. Ce niveau de pression est suffisamment élevé pour induire une déformation plastique ou pour causer une rupture locale dans l'échantillon. Les observations en MEB des surfaces exposées montrent que ce type de pulses crée des impacts de tailles et de formes variables. Les impacts isolés apparaissent généralement sous forme de creux ou de cratères. Par définition, le creux est la partie concave de la zone d'impact ayant subi une déformation plastique sans atteindre la limite de la rupture. Le cratère est un creux où la déformation atteint la limite de la rupture et il est entouré d'une zone de déformation plastique contenant des fissures. La micrographie de la figure la correspond à l'état de surface d'un échantillon exposé $90 \mathrm{~min}$ à l'attaque de cavitation. Elle montre l'aspect et la distribution des creux et des cratères sur la face du monocristal de cuivre. Au début de l'essai, le matériau étant ductile, absorbe une partie de l'énergie d'impact en se déplaçant plastiquement. Par conséquent, la perte de la matière dans cette période reste très faible. Au fur et à mesure que le matériau s'écrouit, le déplacement ductile devient plus difficile et son rôle d'amortisseur diminue. Ceci favorise la rupture, la nucléation et la propagation des fissures et augmente la vitesse de la perte de masse. L'exemple de la figure $1 b$ illustre l'état de surface dans cette période où la déformation est encore hétérogène. A la fin de cette période, la surface exposée est entièrement écrouie et les couches superficielles sont pratiquement érodées. La surface présente une rugosité relativement uniforme ( $f$ ig. $l c$ ) et l'érosion entre dans un stade stationnaire. Dès ce stade, l'état de surface reste pratiquement inchangé et le dégât pénètre dans le volume. Les sous-structures de déformation sont développées à leur niveau maximum et correspondent à celles de larges déformations. La coupe transversale d'un échantillon correspondant à ce stade (fig. Id) montre la recristallisation dynamique qui s'étend à une profondeur de $50-60 \mu \mathrm{m}$.

\section{Aspects de la déformation de surface}

Lors d'érosion de cavitation, le caractère dynamique et localisé des pulses de contrainte provoque une réponse des matériaux qui est différente de celle observée sous la déformation volumique et sous le chargement quasistatique. C'est pourquoi il nous a paru intéressant d'effectuer des observations détaillées de la déformation sur la surface attaquée ainsi que sur des plans cristallographiques normaux à la surface.

Pour des échantillons exposés quelques minutes, sur lesquels on observe des impacts isolés, aucune trace de glissements ou de macles n'est visible dans les régions immédiates des impacts. D'une manière générale, la déformation semble être ductile et le déplacement visqueux. Ceci est vérifié sur des différents plans cristallographiques du type (100), (110), (111) et (112) sur lesquels il y a des vecteurs de Burguers qui font apparaître des traces. Autrement dit, la déformation induite par les impacts de cavitation reste assez faible ou assez homogène pour faire apparaître des traces de déplacement. Cependant des traces de déformation longues mais fines apparaissent même dans des endroits non impactés sur la surface exposée. Il est évident que le nombre de ces lignes augmente et les traces deviennent plus marquées et plus épaisses avec le temps d'exposition (fig. 2a). Cet exemple correspond à un essai de $3 \mathrm{~h}$. On note aisément le caractère discontinu des traces qui témoigne la déviation du glissement sur des plans cristallographiques différents. L'accumulation successive et non uniforme de déformation et le caractère triaxial de la contrainte appliquée peuvent favoriser cet aspect spécifique de la déformation dans les régions qui entourent la zone d'impact.

Les traces de déformation sur des plans normaux à la face érodée sont relativement plus marquées que celles sur la surface d'érosion. Un exemple typique d'aspect des lignes sur une coupe (111) d'un échantillon érodée pendant $1,5 \mathrm{~h}$ selon $<110>$ est présenté à la figure $2 b$. Il illustre la déformation dans une profondeur de $500 \mu \mathrm{m}$ de la surface érodée. L'analyse détaillée des traces de déformation montre, qu'en plus des macles de déformation et des systèmes de glissement compact du type (111), il existe des traces de glissements appartenant aux systèmes non compacts du type (100), (115), (112) et (210). La figure $2 d$ montre l'état de déformation sur une face érodée (110) et les deux systèmes visibles correspondent aux plans de glissement du type (1II) et (112). Par contre, la figure $2 c$ est la micrographic optique d'une coupe (110) d'un échantillon expori selon le plan (111). On constate quatre systèmes de glissement actifs correspondant aux plans cristallographiques : (115), (131), (201) et (211).

L'apparition systématique des macles de déformation et les systèmes non compacts de glissement peuvent être attribués à la déformation sévère et rapide sous forte contrainte induite par les impacts.

\section{Sous-structures de déformation}

L'évolution des microstructures de déformation d'un échantillon érodé dépend d'une part du temps d'exposition et d'autre part de la distance à la surface. Les premières couches avoisinantes de la surface sont sévèrement écrouies jusqu'à pratiquement la rupture tandis que dans des couches plus profondes l'écrouissage diminue continuellement en s'éloignant de la surface. Par conséquent, les sous-structures relatives sont de moins en moins développées.

Afin de mettre en évidence le changement dans la structure le long de la direction des impacts, la surface érodée a été couverte par un dépôt électrolytique de cuivre. Ensuite, les lames de $0,5 \mathrm{~mm}$ d'épaisseur ont été prélevées dans la zone d'érosion selon les plans cristallographiques désirés au moyen d'usinage chimique. L'amincissement de ces échantillons s'effectue à des distances variées de la surface d'érosion. Nous résumons ci-dessous l'ensemble des obser- 

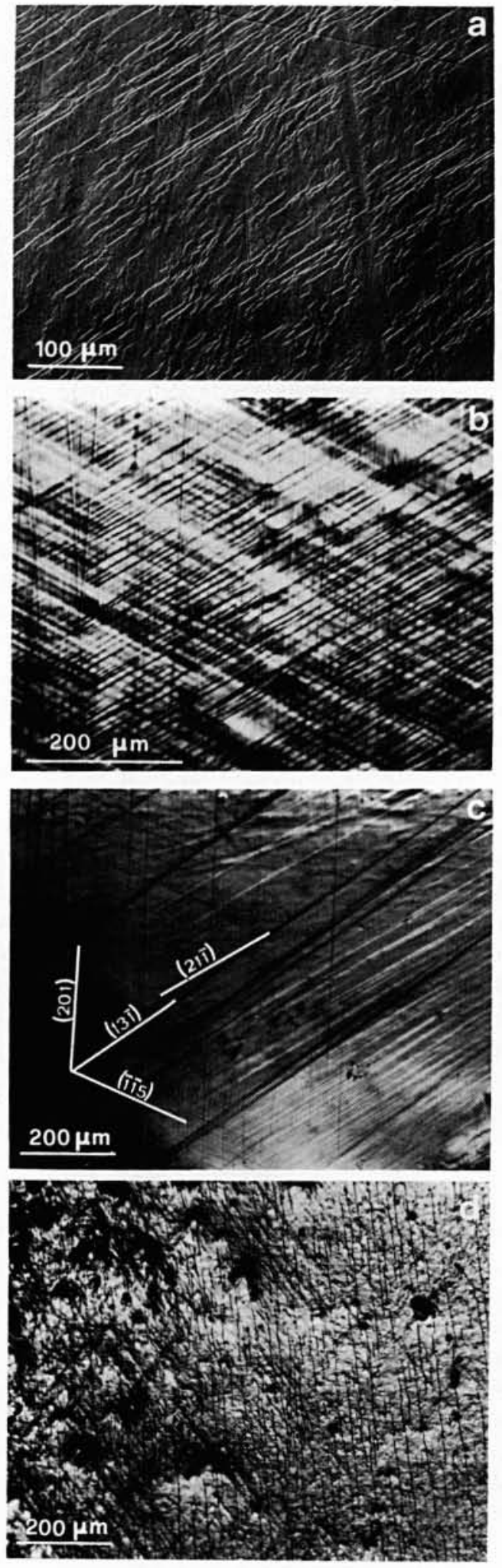

2. Aspects de la déformation sur la surface; a) Lignes de glissement discontinu autour de la zone d'impacts sur la surface exposée $3 \mathrm{~h}$. b) Traces de déformation sur un plan cristallographique normal à la surface. c) Traces de glissement compact et non compact sur un plan (110) normal à la face attaquée. d) Exemple de lignes de glissement non compact sur la face érodée.
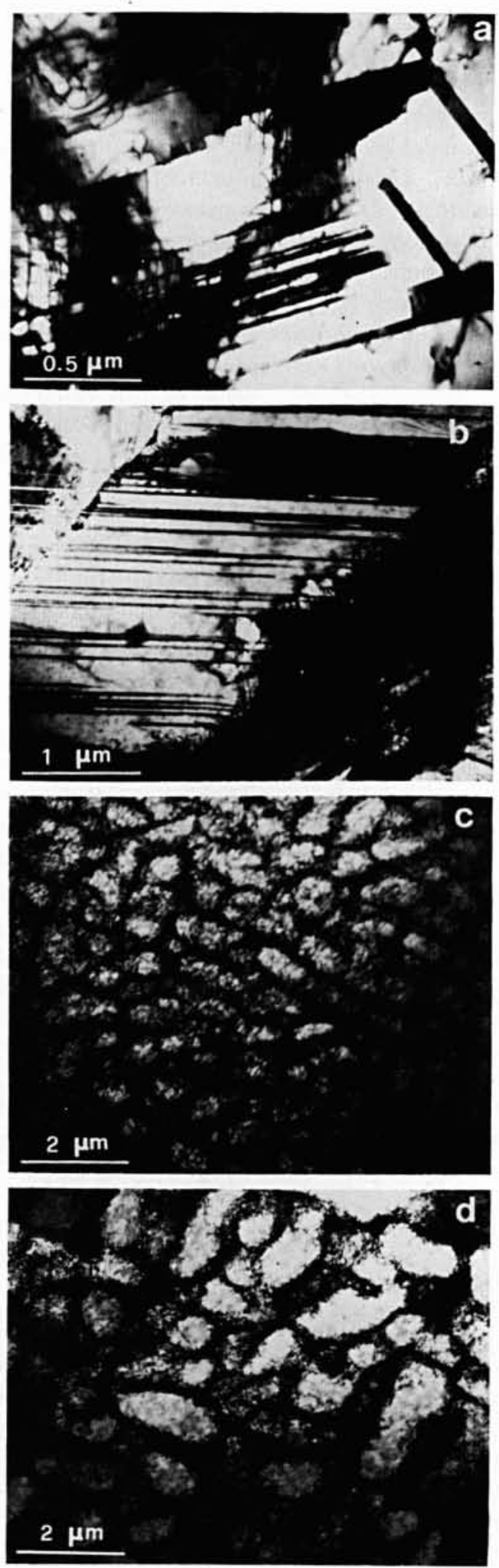

3. Sous-structures de déformation induites par le collapse de cavitation. a) Macles de déformation à $\sim 3-4 \mu \mathrm{m}$ de la surface. b) Microstructures de déformation $\sim 5 \mu \mathrm{m}$ de la surface. c) Cellules de dislocations à $\sim 20 \mu \mathrm{m}$ de la surface. d) Cellules de dislocation à $250 \mu \mathrm{m}$ de la surface. 
vations effectuées en microscopie électronique à transmission.

Dans le cas d'un échantillon fortement érodé, la microstructure est constituée de macles de déformation et de dislocations. Les macles sont la sous-structure dominante jusqu'à une profondeur d'environ $30 \mu \mathrm{m}$, mais s'étendent jusqu'à $150-200 \mu \mathrm{m}$ de la surface. Dans des couches adjacentes de la surface érodée, on observe des blocs maclés de 5-8 $\mu \mathrm{m}$ de diamètre, qui eux-mêmes sont constitués de micro-macles d'orientations différentes (fig. $3 a$ ). Plus loin apparaissent des bandes de macles d'une épaisseur de $0,2 \mu \mathrm{m}$ espacées de $\sim 1 \mu \mathrm{m}$. D'une manière générale, dans ces régions on observe trois systèmes de macles. On distingue ces trois systèmes soit à la fois dans une même profondeur soit des bandes successives dans des profondeurs différentes. Un exemple de ce type de macles est présenté sur la figure $3 b$. Il a été observé dans un échantillon érodé $100 \mathrm{~h}$ selon [110]. On constate que la densité de dislocations dans les régions maclées est inférieure à celle dans les régions où les macles ne se produisent pas. La nucléation, la croissance et la propagation des macles et leur interaction avec les dislocations dans la région de transitions sont des mécanismes assez complexes et ne figurent pas dans le cadre de la présente communication.

Les macles dans des profondeurs de 150-200 $\mu \mathrm{m}$ sont plus fins mais plus longs. Ceci laisse penser qu'elles ont probablement été formées plus près de la surface puis étendues dans la profondeur par l'écrouissage progressif le long de l'expérience. La présence de micro-macles dans ces profondeurs, contrairement aux macles proches de la surface ne semble pas avoir affecté la densité de dislocations. Cependant leur apparition et leur interaction semblent dissiper une quantité considérable de la contrainte interne du matériau. L'interaction de deux macles observée en microscopie électronique ả haute résolution est montrée dans la figure 4 . On constate une perturbation très nette du réseau aux environs des régions d'intersection qui témoigne la présence d'une très forte contrainte.

Quant aux dislocations, elles ont une structure cellulaire (fig. $3 c, d$ ). Leur taille est de l'ordre de $0,4-0,5 \mu \mathrm{m}$ au voisinage de la surface et augmente jusqu'à $\sim 20 \mu \mathrm{m}$ à une profondeur d'environ $1,2 \mathrm{~mm}$. La taille de $0,4-0,5 \mu \mathrm{m}$ semble être la taille critique minimale pour les cellules. Une fois cette taille minimale est atteinte, la continuation du chargement provoque le maclage plutôt que réduire les tailles de cellules. Plusieurs de ces cellules forment un bloc dont la taille varie de $2-3 \mu \mathrm{m}$ à $6-8 \mu \mathrm{m}$. La désorientation entre les cellules d'un bloc est de l'ordre de $1-2^{\circ}$. Par contre, la désorientation entre les blocs de cellules peut atteindre $5-6^{\circ}$.

Adresse des auteurs

Messieurs M. Maamouri et A. Karimi

Institut de génie atomique. EPFL

CH-1015 Lausanne (Suisse)

Tël. : 19/41.21.47.33.95

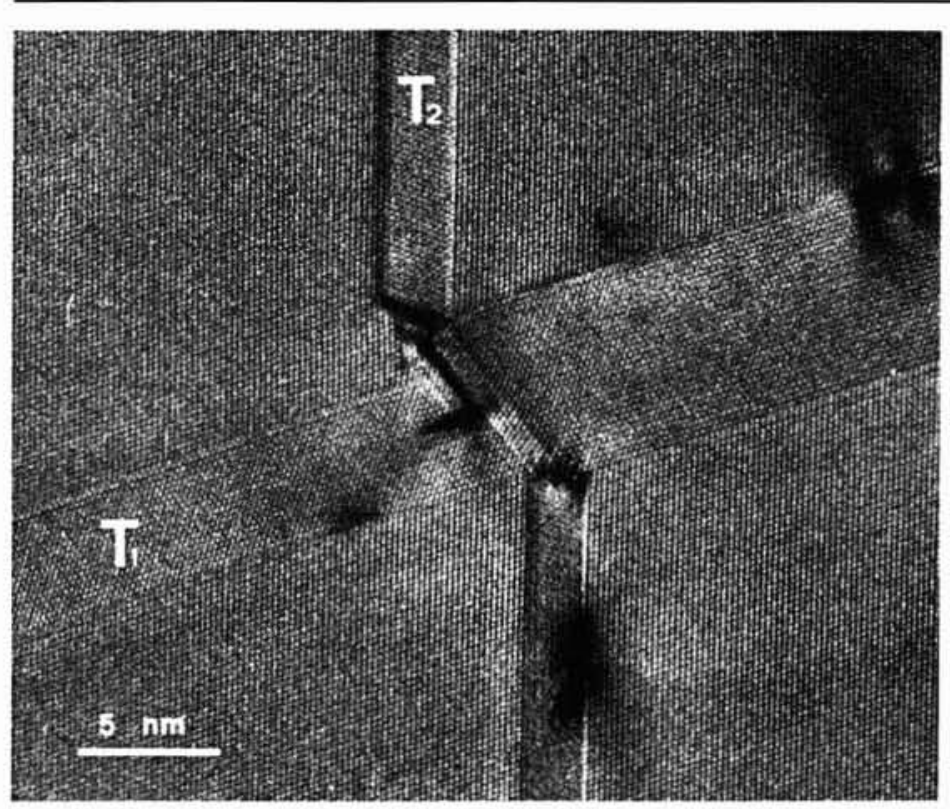

4. Cliché de microscopie électronique à haute résolution montrant l'interaction de deux micro-macles dans le cuivre.

\section{Références}

K.C. ANT(M) W.L. SilenCE. - "The effect of Composition and Microstructure on Cavitation Erosion Resistance ", in Proc. 5 th Int. Conf. on Erosion by Solid and Liquid Impacts, Farnborough, UK, Royal Aircraft Establishment, 1979, p. 67, 1-8.

F. Avellan, A. Karimi, - «Dynamics of Vortex Cavitation involved in the Erosion of Hydraulic Machines ", in Proc. 7th Int. Conf. on Erosion by Liquid and Solid Impacts, J.E. Field, J.P. Dear, eds., 1987, p. 25, 1-8.

T.H. Blewitt, R.R. Coltman, J.K. Redman. - «Low Temperature Deformation of Copper Single Crystals ", J. Appl. Phys. (1957), 6 (28), pp. 651-660.

P.S. Follansbee, D. BanerjeE, J.C. Will.iams. - « Localized Deformation and Fracture due to low Velocity Particulate Erosion of Ductile Metals by spherical Particulates ".

C.J. Heathcock, B.E. Protheroe, A. Ball. - Wear (1982), 80, pp. 311-327.

L.K. Ives, A.W. RUFF. - «Electron Microscopy Study of Erosion Damage in Copper ", in Erosion: Prevention and Useful Applications, ASTM 664, W.F. Adler Ed. ASTM, 1979. pp. 5-35.

A. Karimi, F. Avellan. - "Comparison of Erosion Mechanisms in Different Types of Cavitation ", Wear (1986), 113, pp. $305-322$.

S. Mahajan, D.E. BARry, B.L. EYre. - "A Thin Twin and its Interaction with a Coherent Twin Boundary in Copper ", Phil. Mag. (1969), pp. 43-52.

R.N. Wright, D.E. Mikkola. - Mater. Sci. Eng. (1976), 26, pp. 263-268. 
M. CHIEM : Cet exposé plus métallurgiste amène-t-il des questions?

M. WALDURA: Je pensais que l'érosion de cavitation résultait de successions d'efforts et donc de fatigue. Or, vous avez parlé de dislocations, de glissements et de déformation plastique sans jamais évoquer les particularités des sollicitations cycliques ?

M. MAAMOURI: La cavitation n'est pas à comparer à des essais de fatigue, car les matériaux sont très déformés jusqu'à apparition des cellules. Il faudrait faire des essais avec des matériaux déjà fatigués.

M. KARIMI: La philosophie est que l'érosion est un problème en tant que tel qu'il n'y a pas lieu de rattacher à un autre mécanisme de destruction, telle la fatigue, même s'il y a, dans les deux cas accumulation des dégâts.

Les stries de fatigue sont un aspect macroscopique des déformations dues à l'application de contraintes cycliques et ne suivent généralement pas l'orientation cristallographique.

Dans le cas qui nous intéresse, les marches sur la surface sont des bandes de déformation, c'est-à-dire des bandes de glissement et des mâcles, qui ont une origine cristallographique. Ces bandes sont dues au déplacement d'une partie du cristal par rapport l'autre qui n'a pas commune mesure avec l'apparition des stries.

M. CHIEM : Le terme de fatigue ne convient pas dans ce contexte. A partir des photographies Cranz-Schardin on peut constater qu'il s'agit de chocs répétés.

On peut dire que les microstructures représentent la réponse du matériau à ce genre de sollicitation. Les auteurs ont montré les sollicitations et les mâcles.

Si une grosse mâcle se produit, il y a, à la pointe de la mâcle une zone de concentration de contraintes conique. On peut voir, à l'intérieur de cette zone des micromâcles alignées selon des directions préférentielles.

Les deux grosses mâcles peuvent, soit se propager et se relier en interagissant avec ces micromâcles, ou alors s'éliminer pour disparaître. On trouvera des dislocations autour de cette zone.

Il existe un seuil critique de formation des mâcles, proportionnel à $1 / n$, où $n$ est la concentration des contraintes. Ce seuil est à 2 composantes:

- La première composante est directement liée au mâclage. - La seconde est liée au glissement-dislocation.

Cela donne un compromis, fonction de l'état de contrainte. Quand l'impact vient directement d'en haut, l'intensité des coups est plus importante, d'où une forte concentration de contraintes et formation de mâcles. Plus loin en profondeur, il y a glissement des dislocations, ce qui donne des formations particulières.

M. MAAMOURI : Ces cellules se mettent par petits groupes pour former le mâclage. Au cours du processus, on a, d'abord une déformation très locale, puis apparition de grosses cellules, qui deviennent de plus en plus petites et sont finalement remplacées par des mâcles.

M. CHIEM : Cette évolution correspond à l'équation que j'ai indiquée. La mâcle est déjà une forme d'empilement.

M. CHINCHOLle: Lorsque des morceaux s'en vont, avez-vous une idée de leur diamètre? Je parle des chocs de cavitation et pas d'incubation. Un rayon de l'ordre de $1 \mu$ est-il possible?

M. KARIMI : La taille des particules après érosion peut aller, par exemple, jusqu’à $10 \mu$ dans l'acier inoxydable 316 et jusqu'à 30 $40 \mu$ dans le laiton.

M. DOREY: Les impacts font en général $40 \mu$. L'effet métallurgique semble aller beaucoup plus profond.

$\mathrm{Ne}$ pensez-vous pas qu'il puisse y avoir, d'une part un effet d'onde et d'autre part l'implosion locale de petites structures issues du vortex?

M. MAAMOURI: Ce que l'on vient d'observer en profondeur était bien localisé. Nous considérons que, tant que la surface n'est pas fortement érodée, nous sommes toujours en période d'incubation. Parfois, un seul impact est suffisant pour arracher de la matière, mais ce n'est pas vraiment de l'érosion.

M. COLAS: Avez-vous fait des analogies métallurgiques pour voir si les dislocations que vous avez mises en évidence peuvent être reproduites en essais métallurgiques? A-t'on une idée de la simulation qu'il faut faire pour avoir les mêmes effets?

M. MAAMOURI: La multiplication des dislocations par la déformation est un phénomène connu et admis. Nous n'avons pas fait d'essais de ce genre. Des structures semblables sont fréquemment observées lors des essais de rupture dynamique et d'impact de projectiles.

M. CHIEM : La valeur que vous demandez dépend des systèmes. Elle est au niveau du monocristal anisotrope. La contrainte théorique pour le système le plus actif est de l'ordre de 200 à $300 \mathrm{MPa}$.

M. KARIMI: L'intensité des chocs varie de $50 \mathrm{MPa}$ à 2000 ou $3000 \mathrm{MPa}$. Pour les conditions de travail présentées ici, on obtient une moyenne de $500 \mathrm{MPa}$ sur 472 pulses.

M. DESCLAUX : Concernant le tout début de l'érosion de cavitation, il semble que la surface soit légèrement ondulée. Avez-vous des explications? est-ce uniquement un problème de surface?

M. KARIMI : L'ondulation de la surface est due au caractère localisé et aléatoire des impacts d'une part, et d'autre part à la propriété du matériau, par exemple les joints de grain sont plus durs que les grains eux-mêmes.

Certains impacts sont peu puissants et conduisent à une déformation plastique. D’autres plus puissants peuvent créer une rupture.

M. CHINCHOLLE: Si l'on trace un diagramme donnant la perte de masse en fonction du nombre de chocs, on constate qu'il y a d'abord incubation, sans perte de poids, puis que l'érosion augmente de façon linéaire.

M. HEUZÉ: Le problème concerne les ondes de choc et les micro-jets. Les ondes de choc déformeraient plastiquement la surface en donnant un aspect ondulé, tandis que les micro-jets conduiraient à des empreintes plus profondes mais plus petites.

M. AVELLAN : On a montré qu'il y a plusieurs collapses, et que les phénomènes sont très compliqués. Plus les cavités sont loin de la paroi, moins elles sont agressives, d'où la différence constatée des dégâts.

Je n'ai par contre jamais vu de micro-jet. Ceci mériterait des études complémentaires.

M. CHIEM : De toute façon, on doit créer une onde de choc sur le matériau comme l'ont montré divers travaux.

M. AVELLAN: Il y a au moins 1,5 fois plus d'impacts que de tours d'obturateur.

M. LECOFFRE : Ceci est dû au fait qu'il y a plusieurs collapses par tour d'obturateur du fait des rebonds successifs. Avec les réglages de M. Heuzé, il y a en général 2 collapses. Avec ceux de Lausanne, il se peut qu'il y en ait trois ou même plus. Ce qui est certain, c'est que le premier est le plus agressif. Il n'est donc pas étonnant que l'on ait un nombre d'impacts supérieur au nombre de tours.

Je ferai également remarquer, d'un point de vue plus général qu'il avait été envisagé d'analyser le problème de la réponse élasto-plastique des matériaux. Cette suggestion reste valable, car il semble qu'il y ait un certain manque de publications sur le couplage fluide-structure sous l'effet d'un impact unique.

M. HEUZE: Sur quelle épaisseur la recristallisation a-t-elle été observée? S'il y a recristallisation, la dureté doit diminuer. Vous auriez dû observer une chute de dureté sur les 50 premiers microns.

M. MAAMOURI: Nous nous trouvions dans un cas oủ la dureté augmente. Ce comportement dépend en effet de la taille des grains. Il y a une influence de l'écrouissage. 